\title{
Complete aortic sac shrinkage following endovascular repair of ruptured abdominal aortic aneurysm (REVAR)
}

\author{
Mohamed Elsherif ${ }^{1}$, Wael Tawfick ${ }^{1}$, Murali Subramaniam ${ }^{1}$ and Sherif Sultan ${ }^{1,2}$ \\ ${ }^{1}$ Western Vascular Institute, Department of Vascular and Endovascular Surgery, Galway University Hospital, National University of Ireland, Galway, Ireland \\ ${ }^{2}$ Department of Vascular and Endovascular Surgery, Galway Clinic, Ireland
}

\begin{abstract}
We report on 6 months follow up of an endovascular repair of a ruptured abdominal aortic aneurysm (REVAR). An Aorto-Bi-iliac device was used. At 6 months, the aortic sac showed complete shrinkage back to a normal size. This case illustrates the remodelling that happens following endovascular management of abdominal aortic aneurysms (AAA).
\end{abstract}

\section{Case report}

A 59 year old gentleman with a background history of hypertension and hyperlipedaemia, presented to the emergency department with abdominal and chest pain. He was confused and haemodynamically unstable. His blood pressure at presentation was 70/40 His pulse was 125 , on examination his abdomen was tender centrally and in the left flank. A fast scan revealed asizeable abdominal aortic aneurysm. Patient underwent a CT scan angiogram on route to the operating theatre. The CT scan confirmed a rupturedabdominal aortic aneurysm (AAA). It was $5.8 \mathrm{~cm}$ at maximum diameter. There was a large retroperitoneal haematoma on the left side. The patient had bilateral common iliacsaccular artery aneurysms, measuring $3.5 \mathrm{~cm}$ on the right and 2.5 $\mathrm{cm}$ on the left (Figures 1). The anatomy of the aneurysm was suitable for endovascular repair of the ruptured abdominal aortic aneurysm (REVAR).

The patient underwent a REVAR, using an Endurant II, AortoBi-Iliac device (Medtronic Vascular). Following the exclusion of the aneurysm, the patient became haemodynamically stable. He was transferred to the intensive care unit (ICU).

The procedure was complicated with acute abdominal compartmental syndrome. This was associated with acute kidney

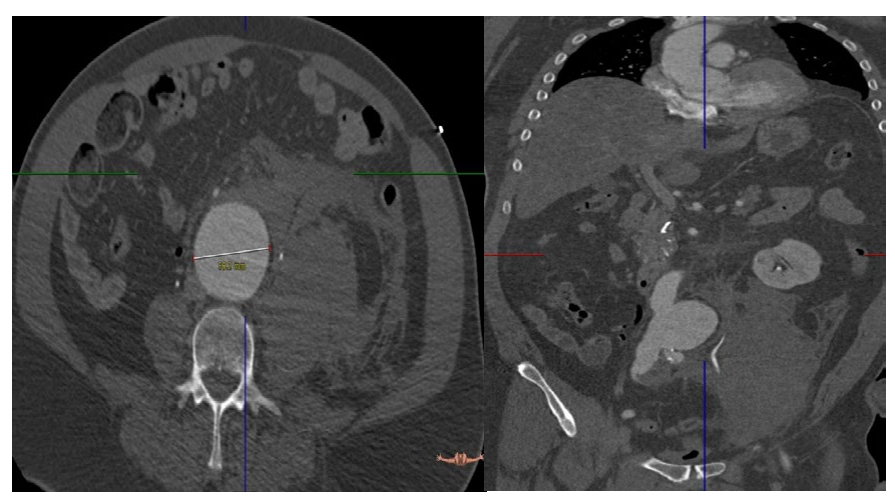

Figure 1. Pre-op CT showed rupture AAA $5.8 \mathrm{~cm}$ with retroperitoneal hematoma. injury, high inotrope support requirements and progressively difficult ventilation. He underwent a decompression laparostomy with evacuation of the retroperitoneal hematoma. Negative pressure vacuum therapy was used to cover the laparostomy. The patient had his abdominal wound closed by secondary intention 2 weeks later. $\mathrm{He}$ required temporary sessions of hemodialysisduring his 2 week ICU stay. His kidney functions gradually improved and he was discharged home on day 60 post-operatively.

At 6 months follow up the patient was generally well. His follow up CT scan angiogram revealed a complete remodelling of the aorta, with total shrinkage of the aneurysmal sac (Figures 2). There was no residue

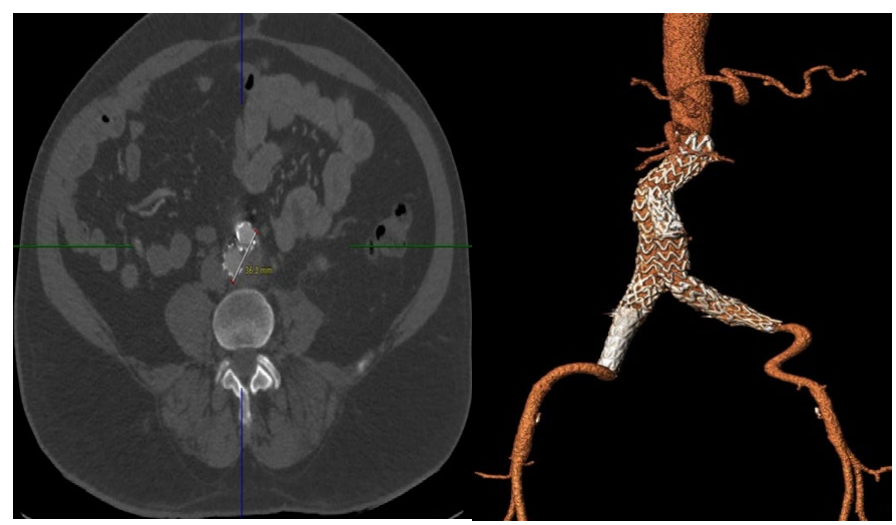

Figure 2. 6 months Follow-up CT demonstrated total remodelling of aortic sac post EVAR with patent EVAR graft.

Correspondence to: Prof. Sherif Sultan, Professor of Vascular and Endovascular Surgery,Western Vascular Institute, Department of Vascular and Endovascular Surgery,University Hospital Galway, National University of Ireland, Galway, Ireland, Tel: +35391 720121; Fax: +35391720122; E-mail: sherif.sultan@hse.ie

Key words: sac, shrinkage, AAA repair, REVAR

Received: October 15, 2015; Accepted: November 18, 2015; Published: November 21, 2015 
of aneurysm in this patient's abdominal aorta. The left common iliac aneurysm completely remodelled, with no trace of the aneurysm. His right common iliac aneurysm appeared to be completely excluded, with no signs of endoleak, however, it had not yet remodelled completely. The maximum diameter of the right common iliac aneurysm was now $2.5 \mathrm{~cm}$.

\section{Discussion}

This case reports on one of the quickest cases of AAA sac regression following endovascular repair of abdominal aortic aneurysm. The sac regression occurred in less than 6 months. Malian et al. [1] in one of the few reports to go beyond 1 year of follow-up, proposed that most of the regression of the AAA sac occurred in the first year with minimal changes thereafter. Rhee et al. [2] concluded that a complete collapse of the AAA is also much more likely during the second year of followup when compared with the first. In addition, a small percentage of patients who had not yet shown a reduction in size by 12 months will show regression at the 2-year follow up. Our case, however, revealstotal remodelling of aortic sac aneurysm, with complete shrinkage of the aortic sacwithin 6 months.

The definition of significant reduction in size was arbitrarily set at a minimum of $0.5 \mathrm{~cm}$ [3]. Large-scale analyses of interobserver and intraobserver variability in evaluating AAA size from CT scans has shown that a 5-mm threshold would represent an actual change in the aneurysm rather than measurement error [3]. The use of the minor diameter on the axial slices also ensures the reduction in measurement variability due to tortuosity of the AAA and positioning of the patient.

The relationship between sac pressure and changes in sac diameter over time is becoming clearer. Dias et al. [4] demonstrated that shrinking aneurysms were associated with low sac pressures and enlarging aneurysms were associated with elevated sac pressures. This was confirmed by Sonesson et al. [5] who performed direct translumbar puncture in patients after EVAR. He showed that the mean intrasac pressure diminished to $20 \%$ of the mean arterial pressurein patients with sac shrinkage and no evidence of endoleak.

The concept of AAA sac regression after endoluminal repair appears intuitive. When the arterial pressure is redirected through the endoluminal device, the AAA sac should collapse around the graft $[6,7]$. However, this is not always the case. When AAAs are excluded by simple ligation and bypass graft, leaving patent lumbar arteries and the inferior mesenteric artery, a number of these patients can continue to expand and even rupture [3]. Another proposed theory implicates a neointimal hyperplastic reaction over the endograft surface, which in turn causes a fibrotic shrinkage of the AAA sac. This fibrotic process has not been confirmed clinically but is reported to occlude small arterioles and even lumbar arteries, helping obliterate the sac.It is rare to find a case with this total remodelling in a very short time post REVAR. We presume the cause to be multifactorial (the choice of the device, the device's size, the sac pressure as well as occluded lumber arteries).

\section{Conclusion}

REVAR is becoming more mainstream and is proving to be durable and effective. Further studies are required to identify factors that expedite sac remodelling post EVAR.

\section{References}

1. Malina M, Länne T, Ivancev K, Lindblad B, Brunkwall J (1998) Reduced pulsatile wall motion of abdominal aortic aneurysms after endovascular repair. J Vasc Surg 27: 624-631. [Crossref]

2. Rhee RY, Eskandari MK, Zajko AB, Makaroun MS (2000) Long-term fate of the aneurysmal sac after endoluminal exclusion of abdominal aortic aneurysms. $J$ Vasc Surg 32: 689-696. [Crossref]

3. Lawrence DD Jr, Charnsangavej C, Wright KC, Gianturco C, Wallace S (1987) Percutaneous endovascular graft: experimental evaluation. Radiology 163: 357-360. [Crossref]

4. Dias NV, Ivancev K, Malina M, Resch T, Lindblad B, et al. (2004) Intra-aneurysm sac pressure measurements after endovascular aneurysm repair: differences between shrinking, unchanged, and expanding aneurysms with and without endoleaks. J Vasc Surg 39: 1229-1235. [Crossref]

5. Sonesson B, Dias N, Malina M, Olofsson P, Griffin D, et al. (2003) Intra-aneurysm pressure measurements in successfully excluded abdominal aortic aneurysm after endovascular repair. J Vasc Surg 37: 733-738. [Crossref]

6. Harris P, Brennan J, Martin J, Gould D, Bakran A, et al. (1999) Longitudinal aneurysm shrinkage following endovascular aortic aneurysm repair: a source of intermediate and late complications. $J$ Endovasc Surg 6: 11-16. [Crossref]

7. Malina M, Länne T, Ivancev K, Lindblad B, Brunkwall J (1998) Reduced pulsatile wall motion of abdominal aortic aneurysms after endovascular repair. J Vasc Surg 27: 624-631. [Crossref]

8. Shah DM, Chang BB, Paty PS, Kaufman JL, Koslow AR, et al. (1991) Treatment of abdominal aortic aneurysm by exclusion and bypass: an analysis of outcome. $J$ Vasc Surg 13: 15-20. [Crossref]

Copyright: (C2015 Elsherif M. This is an open-access article distributed under the terms of the Creative Commons Attribution License, which permits unrestricted use, distribution, and reproduction in any medium, provided the original author and source are credited. 